\title{
ONE-PARAMETER GROUPS OF ISOMETRIES ON HARDY SPACES OF THE TORUS
}

\author{
BY \\ EARL BERKSON AND HORACIO PORTA ${ }^{(1)}$
}

\begin{abstract}
The strongly continuous one-parameter groups of isometries on $H^{p}$ of the torus $(1<p<\infty, p \neq 2)$, as well as their generators, are classified and concretely described.
\end{abstract}

0. Introduction. The purpose of this paper is to investigate the strongly continuous one-parameter groups of isometries of the $H^{p}$ spaces of the torus, for $1 \leqslant p<\infty, p \neq 2$. We shall characterize and classify all such groups, as well as describe their infinitesimal generators.

The space $H^{p} \quad(1 \leqslant p<+\infty)$ of the torus $C^{2}$ is defined (see [5]) as the subspace of complex $L^{p}\left(C^{2}\right)$ consisting of those functions $f$ whose double Fourier coefficients $c_{k, j}$ vanish if $(k, j) \notin P$, where $P$ is the "positive set" in the character group of $C^{2}, P=\{(m, n): n>0\} \cup\{(m, 0): m \geqslant 0\}$.

The study of one-parameter groups of isometries of $H^{p}\left(C^{2}\right)$ is more involved than its counterpart for the circle $C$ (see [1] and [2] for the latter), because of the lack of symmetry in the roles of the independent variables in $H^{p}\left(C^{2}\right)$.

The crucial fact for our purposes is the following result of Lal and Merrill (throughout what follows, we shall use $(z, w)\left(z=e^{i \theta}, w=e^{i \psi}\right)$ to denote the general point of $C^{2}$ ).

(0.1) THEOREM [5]. If $T$ is a linear isometry from $H^{p}\left(C^{2}\right)$ onto $H^{p}\left(C^{2}\right)$ $(1 \leqslant p<\infty, p \neq 2)$, then there are $\alpha \in C$, a Möbius transformation of the disc $\varphi$, and a measurable function $\sigma: C \rightarrow C$ such that for all $f \in H^{p}\left(C^{2}\right)$ :

$$
(T f)(z, w)=\alpha\left[\varphi^{\prime}(z)\right]^{(1 / p)} f(\varphi(z), \sigma(z) w)
$$

for almost all $(z, w)$.

On the other hand, if $1 \leqslant p<\infty$ and $\alpha, \varphi$ and $\sigma$ are as above, the righthand side of $(0.2)$ defines a linear isometry of $H^{p}\left(C^{2}\right)$ onto $H^{p}\left(C^{2}\right)$.

For $1 \leqslant p<\infty$, if $Z^{p}$ is the closure in $L^{p}\left(C^{2}\right)$ of the polynomials in $z$, it

Presented to the Society, August 20, 1975; received by the editors January 28, 1975.

AMS (MOS) subject classifications (1970). Primary 46E15, 46E30, 47D05. generator.

Key words and phrases. Isometry, Hardy space, torus, Möbius transformation, group,

(1) The work of the authors was supported by a National Science Foundation grant. 
is clear that an operator of the form (0.2) will leave the subspace $Z^{p}$ invariant. Thus, when $p \neq 2$, strongly continuous one-parameter groups of isometries in $H^{p}\left(C^{2}\right)$ can be naturally classified into those whose restriction to $Z^{p}$ is continuous in the uniform operator topology and those whose restriction is not.

In $\S 1$ we consider the groups of the first kind. In particular, we show there that for $1 \leqslant p<\infty, p \neq 2$, the isometric groups on $H^{p}\left(C^{2}\right)$ continuous in the uniform operator topology are the trivial ones, i.e., the groups of the form $\left\{e^{i \rho t} I\right\}$ where $\rho$ is real and $I$ is the identity operator.

The remainder of the paper is devoted to the groups $\left\{T_{t}\right\}$ of the second kind. In $\$ 2$ we study their underlying structure. Each such group has associated with it a family $\left\{\varphi_{t}\right\}$ of conformal maps of the disc and a family $\left\{\sigma_{t}\right\}$ of unimodular measurable functions on $C$. One has in particular, for $s, t$ real,

$$
\varphi_{t+s}(z)=\varphi_{t}\left(\varphi_{s}(z)\right) \quad \text { for } z \in C
$$

and

$$
\sigma_{t+s}(z)=\sigma_{t}\left(\varphi_{s}(z)\right) \sigma_{s}(z) \quad \text { for almost all } z \in C \text {. }
$$

The properties of the group $\left\{T_{t}\right\}$ depend heavily on the nature of the set $S$ of common fixed points, in the extended plane, of the group $\left\{\varphi_{t}\right\} . S$ is known to be: (i) a doubleton set of symmetric points with respect to the circle $C$, (ii) a singleton subset of $C$, or (iii) a doubleton subset of $C$ (see [2]), and correspondingly we shall say that the group $\left\{\varphi_{t}\right\}$ is of type (i), (ii) or (iii).

It turns out that there are a unimodular measurable function $u$ on $C$ and a real constant $\delta$ such that $\left\{\sigma_{t}\right\}$ can be taken in the form

$$
\sigma_{t}=e^{i \delta t} u\left(\varphi_{t}\right) \bar{u}, \text { for } t \text { real. }
$$

If $\left\{\varphi_{t}\right\}$ is of type (ii) or (iii), then $\delta$ can be taken to be zero.

We calculate the infinitesimal generator of $\left\{T_{t}\right\}$ in $\S 3$ for the case $\delta=0$ and in $\S 4$ for the case $\delta \neq 0$.

The following notation will be used throughout. The symbols Z, R, C and $\mathbf{C}_{e}$ will denote, respectively, the set of integers, the real line, the complex plane and the extended plane. We denote the open unit disc $\{z \in \mathrm{C}:|z|<1\}$ by $D$ and the closure of $D$ by $\bar{D}$. For $z \in \mathrm{C}, \operatorname{Re} z$ and $\operatorname{Im} z$ will be the real and imaginary parts of $z$. For $m, n \in \mathrm{Z}, e_{m, n}$ will be the function on $C^{2}$ given by $e_{m, n}(z, w)=z^{m} w^{n}$, and $\lambda$ will be normalized Lebesgue measure on $C$. We denote composition of maps by $\circ$. If $f$ is a function defined on $C^{2}$, and $z_{0}$ (resp., $w_{0}$ ) belongs to $C$, then the $z_{0}$-section (resp., the $w_{0}$-section) of $f$ is the function $f\left(z_{0}, \cdot\right)$ (resp., $\left.f\left(\cdot, w_{0}\right)\right)$.

The authors are indebted to Professor Robert P. Kaufman for valuable comments and suggestions. 
1. Groups whose $Z^{p}$-restriction has a bounded generator. Let $\left\{T_{t}\right\}$ be a strongly continuous group of isometries on $H^{p}\left(C^{2}\right), 1 \leqslant p<\infty$ (if $p=2$, we assume further that each $T_{t}$ has the form (0.2)), such that $\left\{T_{t} \mid Z^{p}\right\}$ is continuous in the uniform operator topology (the vertical bar denotes restriction). Since $Z^{p}$ can be identified with $H^{p}(C)$, it follows from [2, Theorem (2.4)] and Theorem (0.1) above that for each $t \in \mathbf{R}$, there are a unimodular constant $\alpha_{t}$ and a unimodular measurable function $\sigma_{t}$ on $C$ such that:

$$
\left(T_{t} f\right)(z, w)=\alpha_{t} f\left(z, \sigma_{t}(z) w\right) .
$$

Taking $f$ in (1.1) to be $e_{0,0}$ we conclude that $\alpha_{t}$ is uniquely determined for each $t$, and that $t \mapsto \alpha_{t}$ is a continuous character of $\mathbf{R}$; hence there is a constant $\rho \in \mathbf{R}$ such that $\alpha_{t}=e^{i \rho t}$ for $t \in \mathbf{R}$. By taking $f$ in (1.1) to be $e_{0,1}$, we see that for each $t, \sigma_{t}$ is uniquely determined up to equality almost everywhere, and that: (i) for $s, t \in R, \sigma_{t+s}(z)=\sigma_{t}(z) \sigma_{s}(z)$ for almost all $z \in C$; (ii) the map $t \mapsto \sigma_{t}$ is continuous from $R$ to $L^{p}(d \lambda)$ (or, equivalently, since the $\sigma_{t}$ are unimodular, from $\mathbf{R}$ to $L^{1}(d \lambda)$ ).

The method on pp. 61 and 62 of [3] shows that in view of (ii) above, we can assume without loss of generality that $\sigma_{t}(z)$ is measurable in $(t, z)$ on $\mathbf{R} \times C$. It follows from (i) above that there is a negligible set $N \subset C$ such that if $z \notin N$, $t \mapsto \sigma_{t}(z)$ is measurable and

$$
\int d s \int\left|\sigma_{s+t}(z)-\sigma_{s}(z) \sigma_{t}(z)\right| d t=0
$$

Thus, for each $z \notin N$, there is a negligible set $N_{z} \subset \mathrm{R}$ such that if $s \notin N_{z}$, then

$$
\int\left|\sigma_{s+t}(z)-\sigma_{s}(z) \sigma_{t}(z)\right| d t=0
$$

Now, if $z \notin N$ and $s \notin N_{z}$, there is a negligible set $N_{z, s} \subset \mathbf{R}$ such that

$$
\sigma_{t+s}(z)=\sigma_{t}(z) \sigma_{s}(z) \text { for } t \notin N_{z, s} .
$$

From (1.2) we get

$$
\int_{s}^{s+h} \sigma_{t}(z) d t=\sigma_{s}(z) \int_{0}^{h} \sigma_{t}(z) d t
$$

for $z \notin N, s \notin N_{z}$ and any $h \in \mathbf{R}$.

Now fix $z \in C \backslash N$ and choose $h$ such that $\int_{0}^{h} \sigma_{t}(z) d t \neq 0$. Let

$$
F_{z}(u)=\int_{u}^{u+h} \sigma_{t}(z) d t / \int_{0}^{h} \sigma_{t}(z) d t, \quad u \in \mathbf{R} .
$$

Then $F_{z}$ is continuous on $\mathbf{R}$ and $\sigma_{s}(z)=F_{z}(s)$ for all $s \in \mathbf{R} \backslash N_{z}$. In particular $\left|F_{z}\right| \equiv 1$. 
It follows from (1.2) that if $s \notin N_{z}$, then $F_{z}(t+s)=F_{z}(t) F_{z}(s)$ for $t \notin$ $N_{z, s} \cup N_{z} \cup\left(N_{z}-s\right)$. From continuity of $F_{z}$ and Fubini's Theorem we conclude that $F_{z}$ is a continuous character of $\mathbf{R}$ and so there is a constant $\delta(z) \in \mathbf{R}$ such that

$$
\sigma_{s}(z)=e^{i \delta(z) s} \quad \text { for } s \in \mathrm{RW} \mathrm{z}_{\mathrm{z}}
$$

For $z \in C \backslash N$ and $s \in \mathbf{R}$,

$$
e^{i \delta(z) s}=\lim _{n} n \int_{s}^{s+(1 / n)} e^{i \delta(z) u} d u=\lim _{n} n \int_{s}^{s+(1 / n)} \sigma_{u}(z) d u .
$$

But $\int_{s}^{s+(1 / n)} \sigma_{u}(z) d u$ is measurable in $(s, z)$. Thus, there is a negligible set $Q \subset$ $\mathbf{R}$ such that for $s \in \mathbf{R} \backslash Q, e^{i \delta(z) s}$ is a measurable function of $z$. Let $s_{n} \rightarrow 0$ with $s_{n}>0$ and $s_{n} \notin Q$. Since

$$
i \delta(z)=\lim _{n}\left(e^{i \delta(z) s_{n}}-1\right) s_{n}^{-1},
$$

$\delta(z)$ is a measurable function of $z$.

By (1.3) and Fubini's Theorem, we have that for almost all $s \in \mathbf{R}, e^{i \delta(z) s}$ $=\sigma_{s}(z)$ for almost all $z \in C$. Hence, for almost all $t \in \mathbf{R}$,

$$
T_{t} f=e^{i \rho t} f\left(z, e^{i \delta(z) t} w\right), \quad f \in H^{p}\left(C^{2}\right) .
$$

Since each side of (1.4) separately describes a strongly continuous one-parameter group, we conclude:

(1.5) THEOREM. Let $\left\{T_{t}\right\}$ be a group as in the first sentence of this section. Then there are a real constant $\rho$ and a real-valued measurable function $\delta(\cdot)$ on $C$ such that (1.4) holds for all $t \in \mathbf{R}$. Conversely if $1 \leqslant p<\infty$, then for any such $\rho$ and $\delta(\cdot),(1.4)$ defines such a group $\left\{T_{t}\right\}, t \in \mathbf{R}$.

(1.6) Corollary. Let $p$ satisfy $1 \leqslant p<\infty, p \neq 2$. If $\left\{T_{t}\right\}$ is a one-parameter group of isometries in $H^{p}\left(C^{2}\right)$ continuous in the uniform operator topol$o g y$, then there is a real number $\rho$ such that $T_{t}=e^{i \rho t} I$ for all $t \in \mathbf{R}$.

Proof. Let $\rho$ and $\delta$ be as in Theorem (1.5). Put $S_{t}=e^{-i \rho t} T_{t}$ for $t \in \mathbf{R}$ and let $A$ be the infinitesimal generator of $\left\{S_{t}\right\}$. For each positive integer $n$, the sequence $\left\{k\left[\left(S_{(1 / k)} e_{0, n}\right)-e_{0, n}\right]\right\}_{k=1}^{\infty}$ converges in $H^{p}\left(C^{2}\right)$ to $A e_{0, n}$. It is easy to see that this sequence converges pointwise to in $\delta e_{0, n}$. It follows that $A e_{0, n}=$ in $\delta e_{0, n}$ almost everywhere. Hence $\|\delta\|_{p} \leqslant(1 / n)\|A\|$ for all $n$, so that $\delta=0$ as claimed.

(1.7) THEOREM. Let $\rho$ be a real constant and $\delta(\cdot)$ a measurable real-valued function on $C$. For $1 \leqslant p<\infty$, let $\left\{T_{t}\right\}$ be the group on $H^{p}\left(C^{2}\right)$ defined by

$$
T_{t} f=e^{i \rho t} f\left(z, e^{i \delta(z) t} w\right), \quad f \in H^{p}\left(C^{2}\right) .
$$


Then the domain of the infinitesimal generator $A$ of $\left\{T_{t}\right\}$ consists of those functions $f \in H^{p}\left(C^{2}\right)$ for which there is a $g \in H^{p}\left(C^{2}\right)$ so that for almost all $z$ the following hold:

$$
\delta(z)=0 \text { implies } g(z, w)=0 \text { for almost all } w
$$

$\delta(z) \neq 0$ implies that there is a function $F_{z}$ on $C$ such that $F_{z}\left(e^{i \psi}\right)$ is absolutely continuous for $0 \leqslant \psi \leqslant 2 \pi, f(z, w)=F_{z}(w)$ for almost all $w$ and

$$
g\left(z, e^{i \psi}\right)=\delta(z) d\left(F_{z}\left(e^{i \psi}\right)\right) / d \psi
$$

for almost all $\psi$.

If $f$ and $g$ are as above, then

$$
A f=i \rho f+g \text {. }
$$

Proof. It is clear that without loss of generality we can assume that $\rho=0$.

Assume now that $f$ belongs to the domain $D(A)$ of $A$. Then there is a sequence $\left\{t_{k}\right\}$ of positive real numbers such that $t_{k} \rightarrow 0$, and

$$
\int d \lambda(z) \int\left|t_{k}^{-1}\left(T_{t_{k}} f-f\right)-A f\right|^{p} d \lambda(w)<2^{-k} .
$$

Beppo Levi's theorem in conjunction with (1.11) now gives a negligible set $N \subset$ $C$ such that for $z \notin N$,

$$
\int_{0}^{2 \pi}\left|t_{k}^{-1}\left[f\left(z, e^{i\left(\delta(z) t_{k}+\psi\right)}\right)-f\left(z, e^{i \psi}\right)\right]-(A f)\left(z, e^{i \psi}\right)\right|^{p} d \psi \rightarrow 0 .
$$

Fix an arbitrary $z \in C \backslash N$. If $\delta(z)=0$, clearly from $(1.12)(A f)(z, w)=0$ for almost all $w$. If $\delta(z) \neq 0$, put $h_{k}=\delta(z) t_{k}$, and notice that for $a, b \in[0,2 \pi]$ we have from (1.12):

$$
\int_{a}^{b} h_{k}^{-1}\left[f\left(z, e^{i\left(h_{k}+\psi\right)}\right)-f\left(z, e^{i \psi}\right)\right] d \psi \rightarrow \int_{a}^{b} \delta(z)^{-1}(A f)\left(z, e^{i \psi}\right) d \psi .
$$

Let $a$ be a fixed Lebesgue point of $f\left(z, e^{i \psi}\right.$ ) (as a function of $\psi$ ). Since (1.13) can be rewritten as

$$
\begin{aligned}
h_{k}^{-1} \int_{b}^{b+h_{k}} f\left(z, e^{i \psi}\right) d \psi & -h_{k}^{-1} \int_{a}^{a+h_{k}} f\left(z, e^{i \psi}\right) d \psi \\
& \rightarrow \int_{a}^{b} \delta(z)^{-1}(A f)\left(z, e^{i \psi}\right) d \psi,
\end{aligned}
$$

it follows that for almost all $\psi$, 


$$
f\left(z, e^{i \psi}\right)=\int_{a}^{\psi} \delta(z)^{-1}(A f)\left(z, e^{i v}\right) d v+f\left(z, e^{i a}\right) .
$$

Let $G_{z}(\psi)$ be the absolutely continuous function (of $\psi$ ) on $[0,2 \pi]$ defined by the right-hand side of (1.14). Taking $a=0, b=2 \pi$ in (1.13) gives $\int_{0}^{2 \pi}(A f)\left(z, e^{i \psi}\right) d \psi=0$. It follows that $G_{z}(0)=G_{z}(2 \pi)$. Put $F_{z}\left(e^{i \psi}\right)=G_{z}(\psi)$. Thus $f$ and $A f$ are related as $f$ and $g$ in (1.8) and (1.9).

It is obvious that the set of those $f \in H^{p}\left(C^{2}\right)$ such that there is a $g \in$ $H^{p}\left(C^{2}\right)$ so that (1.8) and (1.9) hold is a linear manifold, and that for such an $f$ the equation $B f=g$ well defines a linear operator $B$ which extends $A$. Since $A$ generates a group of isometries, it follows by [4, VIII. 1.11] that the spectrum of $A$ is a subset of $i \mathbf{R}$. Hence in order to establish that $B=A$, it suffices to show that $B-I$ is one-to-one (see [1, Proof of Theorem (3.2)]). Suppose then that $B f=f$. Then for almost all $z$, we have:

(i) $\delta(z)=0$ implies $f(z, w)=0$ for almost all $w$;

(ii) $\delta(z) \neq 0$ implies that there is an absolutely continuous function of $\psi, F_{z}\left(e^{i \psi}\right)$, such that for almost all $\psi, \delta(z) d F_{z}\left(e^{i \psi}\right) / d \psi=g\left(z, e^{i \psi}\right)=f\left(z, e^{i \psi}\right)$ $=F_{z}\left(e^{i \psi}\right)$.

In case (ii), $F_{z}\left(e^{i \psi}\right)=\int_{0}^{\psi} \delta(z)^{-1} F_{z}\left(e^{i v}\right) d v+F_{z}(1)$, and so we get

$$
\delta(z) \frac{d F_{z}\left(e^{i \psi}\right)}{d \psi}=F_{z}\left(e^{i \psi}\right) \text { for all } \psi .
$$

Hence there is a constant $K_{z}$ such that $F_{z}\left(e^{i \psi}\right)=K_{z} e^{\psi / \delta(z)}$. Setting $\psi=0$ and $\psi=2 \pi$, we conclude that $K_{z}=K_{z} e^{2 \pi / \delta(z)}$. Thus $K_{z}=0$. It follows that $f=0$ almost everywhere.

2. Groups whose $Z^{p}$-restriction has an unbounded generator. For $1 \leqslant p$ $<\infty$, let $\Omega_{p}$ be the set of all strongly continuous one-parameter groups $\left\{T_{t}\right\}$ of isometries of $H^{p}\left(C^{2}\right)$ such that $\left\{T_{t} \mid Z^{p}\right\}$ is not continuous in the uniform operator topology (if $p=2$, we further require that each $T_{t}$ have the form (0.2)).

For a group $\left\{T_{t}\right\} \in \Omega_{p}$, let us write

$$
\left(T_{t} f\right)(z, w)=\alpha_{t}\left[\varphi_{t}^{\prime}(z)\right]^{1 / p} f\left(\varphi_{t}(z), \sigma_{t}(z) w\right) \text { for } f \in H^{p}\left(C^{2}\right) .
$$

We observe that for each $t \in \mathbf{R}, \varphi_{t}=\left(T_{t} e_{1,0}\right) /\left(T_{t} e_{0,0}\right)$ and $\sigma_{t}=\left(T_{t} e_{0,1}\right)$ $\left(T_{t} e_{0,0}\right)^{-1}\left(e_{0,1}\right)^{-1}$. Thus the Möbius transformation of the disc $\varphi_{t}$ in (2.1) is uniquely determined, and $\sigma_{t}$ in (2.1) is determined up to equality almost everywhere on $C$. Application of [2, Theorem (2.4)] to $\left\{T_{t} \mid Z^{p}\right\}$ shows that $\left\{\varphi_{t}\right\}$ is a one-parameter group of Möbius transformations of the disc (i.e., $t \mapsto \varphi_{t}$ is a homomorphism of the additive group of $\mathbf{R}$ into the group, under composition, of all Möbius transformations of the disc such that for each $z \in \bar{D}, t \mapsto \varphi_{t}(z)$ is continuous on $\mathbf{R}$, and some $\varphi_{t}$ is not the identity map). It follows from the 
first paragraph on p. 231 of [1], that $\varphi_{t}^{\prime}(z)$ has a continuous logarithm which enables us to define $\left[\varphi_{t}^{\prime}(z)\right]^{1 / p}$ for $t \in \mathbf{R}, z \in \bar{D}$ so as to be continuous on $\mathbf{R} \times$ $\bar{D}$ and satisfy

$$
\left[\varphi_{t+s}^{\prime}(z)\right]^{1 / p}=\left[\varphi_{t}^{\prime}\left(\varphi_{s}(z)\right)\right]^{1 / p}\left[\varphi_{s}^{\prime}(z)\right]^{1 / p}
$$

for all $s, t \in \mathbf{R}, z \in \bar{D}$. Henceforth we shall standardize $\left[\varphi_{t}^{\prime}(z)\right]^{1 / p}$ in this fashion.

With this understanding, $\alpha_{t}$ in (2.1) is uniquely determined by $t$ and, further, the proof of [1, Theorem (2.1)] shows that $t \mapsto \alpha_{t}$ is a continuous character of $\mathbf{R}$. The group property of $\left\{T_{t}\right\}$ together with strong continuity shows that the family $\left\{\sigma_{t}\right\}$ has the following properties:

$$
\text { for } t, s \in \mathbf{R}, \sigma_{t+s}(z)=\sigma_{t}\left(\varphi_{s}(z)\right) \sigma_{s}(z) \text { for almost all } z \in C \text {; }
$$

$$
\begin{aligned}
& t \mapsto \sigma_{t} \text { is continuous from } \mathbf{R} \text { into } L^{p}(d \lambda) \text { and } \\
& \left|\sigma_{t}(z)\right|=1 \text { for } t \in \mathbf{R}, z \in C .
\end{aligned}
$$

We mention here that, conversely, if $t \mapsto \alpha_{t}$ is a continuous character of $\mathbf{R},\left\{\varphi_{t}\right\}$ is a one-parameter group of Möbius transformations of the disc (with $\left[\varphi_{t}^{\prime}(z)\right]^{1 / p}$ standardized as above), and $\left\{\sigma_{t}\right\}, t \in R$, is a family of functions on $C$ satisfying (2.2) and (2.3), then (2.1) defines a group $\left\{T_{t}\right\}$ in $\Omega_{p}$.

We proceed now to characterize the families $\left\{\sigma_{t}\right\}$ which satisfy $(2.2)$ and (2.3).

As a preliminary step, we take up the notion of orbit, defined as follows.

DEFINITION. Let $\left\{\varphi_{t}\right\}$ be a one-parameter group of Möbius transformations of the disc. For each $z \in C$, the orbit of $z$ (under $\left\{\varphi_{t}\right\}$ ) is $\left\{\varphi_{t}(z): t \in \mathbf{R}\right\}$.

(2.4) THEOREM. If $\left\{\varphi_{t}\right\}$ is of type (i) then the orbit of each $z_{0} \in C$ is $C$.

Proof. This follows readily from the fact (see [2, Theorem (1.10)]) that there are a nonzero real constant $c$ (called the angular velocity of $\left\{\varphi_{t}\right\}$ ) and a Möbius transformation of the disc $\mu$ such that $\varphi_{t}(z)=\mu\left(e^{i c t} \mu(z)\right)$ for all $t \in \mathbf{R}$, $z \in C$.

(2.5) THEOREM. If $\left\{\varphi_{t}\right\}$ is of type (ii) with common fixed point $\alpha$, then for each $z_{0} \in C \backslash\{\alpha\}$, the orbit of $z_{0}$ is $C \backslash\{\alpha\}$. The map $t \mapsto \varphi_{t}\left(z_{0}\right)$ is one-to-one, and $\varphi_{t}\left(z_{0}\right)$ traces out $C \backslash\{\alpha\}$ unidirectionally as $t$ increases.

If $\left\{\varphi_{t}\right\}$ is of type (iii) with common fixed points $\alpha, \beta$, then for each $z_{0} \in$ $C \backslash\{\alpha, \beta\}$, the orbit of $z_{0}$ is the component of $C \backslash\{\alpha, \beta\}$ containing $z_{0}$. The map $t \mapsto \varphi_{t}\left(z_{0}\right)$ is one-to-one, and $\varphi_{t}\left(z_{0}\right)$ traces out this component unidirectionally as $t$ increases. 
Proof. We give the proof for $\left\{\varphi_{t}\right\}$ of type (iii); the proof for the type (ii) case is analogous and will be omitted.

Let $E$ be the component of $C \backslash\{\alpha, \beta\}$ containing $z_{0}$. Clearly the orbit of $z_{0}$ is a connected subset of $E$. Let $L$ be the analytic logarithm on the complement in $\mathbf{C}$ of $\{r \alpha: r \geqslant 0\}$ given by

$$
L(z)=\log |z|+i \arg (z), \quad \arg (\alpha)<\arg (z)<\arg (\alpha)+2 \pi .
$$

Denote by $q$ the invariance polynomial of $\left\{\varphi_{t}\right\}$ (see $[2,(1.6)$ and Theorem (1.7)]). Then we have by $[1$, Theorem (1.5)],

$$
i \frac{d}{d t} \arg \left(\varphi_{t}\left(z_{0}\right)\right)=\frac{d}{d t} L\left(\varphi_{t}\left(z_{0}\right)\right)=\overline{\varphi_{t}\left(z_{0}\right)} q\left(\varphi_{t}\left(z_{0}\right)\right) \neq 0 .
$$

It follows that $d \arg \left(\varphi_{t}\left(z_{0}\right)\right) / d t$ is of constant sign, and so $\arg \left(\varphi_{t}\left(z_{0}\right)\right)$ is strictly monotonic. Let $\eta=\sup \left\{\arg \left(\varphi_{t}\left(z_{0}\right)\right): t \in \mathbf{R}\right\}$. If $\arg \left(\varphi_{t}\left(z_{0}\right)\right)$ is strictly increasing (resp., strictly decreasing), then as $t \rightarrow+\infty$ (resp., $t \rightarrow-\infty), \varphi_{t}\left(z_{0}\right) \rightarrow e^{i \eta}$. Hence, for arbitrary $u \in \mathbf{R}, \varphi_{t+u}\left(z_{0}\right) \rightarrow \varphi_{u}\left(e^{i \eta}\right)$. It follows that $\varphi_{u}\left(e^{i \eta}\right)=e^{i \eta}$. The same reasoning applied to $\inf \left\{\arg \left(\varphi_{t}\left(z_{0}\right)\right): t \in R\right\}$ completes the proof for this case.

(2.6) THEOREM. Let $\left\{\varphi_{t}\right\}$ be of type (ii) or type (iii), and let $\left\{\sigma_{t}\right\}$ be a family satisfying (2.2) and (2.3). Then there is a unimodular measurable function $u$ on $C$ such that for each $t \in \mathbf{R}$,

$$
\sigma_{t}(z)=u\left(\varphi_{t}(z)\right) \overline{u(z)}, \text { for almost all } z \in C .
$$

Proof. By the method of pp. 61 and 62 in [3] we can assume without loss of generality that $\sigma_{t}(z)$ is measurable in $(t, z)$ on $\mathbf{R} \times C$.

Let $S$ be the set of common fixed points of $\left\{\varphi_{t}\right\}$, and let $E$ be a component of $C \backslash S$. A standard application of Fubini's Theorem yields the fact that for almost all $z \in C$,

$$
\sigma_{t+s}(z)=\sigma_{t}\left(\varphi_{s}(z)\right) \sigma_{s}(z) \text { for almost all }(t, s) \in \mathbf{R} \times \mathbf{R} .
$$

Fix a $z_{0} \in E$ so that $\sigma_{t}\left(z_{0}\right)$ is a measurable function on $\mathbf{R}$ and also

$$
\sigma_{t+s}\left(z_{0}\right)=\sigma_{t}\left(\varphi_{s}\left(z_{0}\right)\right) \sigma_{s}\left(z_{0}\right) \text { for almost all }(t, s) \in \mathbf{R} \times \mathbf{R} .
$$

Note that the function $\gamma: t \mapsto \varphi_{t}\left(z_{0}\right)$ maps $R$ bicontinuously onto $E$ and sends sets of measure zero in $\mathrm{R}$ onto sets of measure zero in $E$. Define $u: E \rightarrow$ $C$ as follows:

$$
u(z)=\sigma_{t}\left(z_{0}\right), \quad \text { where } z=\varphi_{t}\left(z_{0}\right)
$$

Clearly $|u| \equiv 1$ on $E$ and from the properties of $\gamma$, it follows that $u$ is measurable on $E$. From (2.7), and Fubini's Theorem, there is a negligible set $A \subset \mathrm{R}$ such 
that for each $t \notin A$, there is a negligible set $N_{t} \subset \mathrm{R}$ with the property that (2.7) holds for $s \notin N_{t}$. Thus for such $t$, s:

$$
u\left(\varphi_{t}\left(\varphi_{s}\left(z_{0}\right)\right)\right) \overline{u\left(\varphi_{s}\left(z_{0}\right)\right)}=\sigma_{t+s}\left(z_{0}\right) \overline{\sigma_{s}\left(z_{0}\right)}=\sigma_{t}\left(\varphi_{s}\left(z_{0}\right)\right) .
$$

Since almost all $z \in E$ are of the form $z=\varphi_{s}\left(z_{0}\right)$ with $s \notin N_{t}$, we conclude that for $t \notin A$,

$$
u\left(\varphi_{t}(z)\right) \overline{u(z)}=\sigma_{t}(z) \text { for almost all } z \in E .
$$

Since the function of $z$ described by each side of (2.8) varies with $t$ continuously in $L^{p}(E),(2.8)$ holds for each $t \in \mathbf{R}$. The proof is complete.

(2.9) THEOREM. Let $\left\{\varphi_{t}\right\}$ be of type (i), and let $\left\{\sigma_{t}\right\}$ be a family satisfying (2.2) and (2.3). Then there are a real constant $\delta$ and a unimodular measurable function $u$ on $C$ such that for each $t \in \mathbf{R}$,

$$
\sigma_{t}(z)=e^{i \delta t} u\left(\varphi_{t}(z)\right) \overline{u(z)}, \text { for almost all } z \in C .
$$

Proof. As in the proof of (2.6) we can take $\sigma_{t}(z)$ to be measurable in $(t, z)$ on $\mathrm{R} \times C$. For fixed $z \in C$, the function $t \mapsto \varphi_{t}(z)$ is no longer one-to-one, but it does map negligible sets of $\mathbf{R}$ onto negligible sets of $C$, and moreover, the inverse image of a negligible set is negligible. Let $c$ be the angular velocity of $\left\{\varphi_{t}\right\}$ and let $\omega$ be the period of $\left\{\varphi_{t}\right\}$ (so that $\omega=2 \pi|c|^{-1}$ ).

From (2.2), we observe that for each $t \in \mathbf{R}$, we have for almost all $z \in C$,

$$
\sigma_{\omega+t}(z)=\sigma_{\omega}\left(\varphi_{t}(z)\right) \sigma_{t}(z) \text { and } \sigma_{t+\omega}(z)=\sigma_{t}(z) \sigma_{\omega}(z) \text {. }
$$

Hence, for almost all $z \in C$,

$$
\sigma_{\omega}\left(\varphi_{t}(z)\right)=\sigma_{\omega}(z)
$$

Using Fubini's Theorem once again, we get that for almost all $z \in C,(2.10)$ holds for almost all $t \in \mathbf{R}$, and consequently there is a unimodular constant $e^{i \mu}, \mu \in \mathbf{R}$, such that $\sigma_{\omega}(z)=e^{i \mu}$ for almost all $z \in C$.

It now follows from (2.2) and induction that for each integer $k$ and $t \in \mathbf{R}$ :

$$
\sigma_{t+k \omega}(z)=e^{i k \mu} \sigma_{t}(z) \text { for almost all } z \in C \text {. }
$$

A further application of Fubini's Theorem allows us to conclude the existence of a negligible set $B \subset C$ such that for $z \notin B$, (2.11) holds for all $k$ and almost all $t \in \mathbf{R}$.

It follows from (2.2) and the foregoing that there are a $z_{0} \in C$ and a negligible set $A \subset \mathrm{R}$ such that

$$
\sigma_{t}\left(z_{0}\right) \text { is measurable on } \mathbf{R} \text {; }
$$




$$
\sigma_{\omega}(z)=\sigma_{\omega}\left(z_{0}\right)=e^{i \mu} \text { for almost all } z \in C ;
$$

(2.14) for each $t \in \mathbf{R} \backslash A$ there is a negligible set $N_{t} \subset \mathrm{R}$ with the property

$$
\sigma_{t+s}\left(z_{0}\right)=\sigma_{t}\left(\varphi_{s}\left(z_{\dot{0}}\right)\right) \sigma_{s}\left(z_{0}\right) \text { for each } s \notin N_{t} .
$$

$$
\sigma_{t+k \omega}\left(z_{0}\right)=e^{i k \mu} \sigma_{t}\left(z_{0}\right) \text { for each integer } k \text { and } t \in \mathbf{R} \backslash A \text {. }
$$

Observe that for all $s, v \in \mathbf{R}, \varphi_{s}\left(z_{0}\right)=\varphi_{v}\left(z_{0}\right)$ if and only if $(s-v)$ is an integral multiple of $\omega$. Let $\gamma(t)=\varphi_{t}\left(z_{0}\right)$ for $t \in \mathbf{R}$. Then $\gamma(A)$ is negligible. Define $u: C \backslash \gamma(A) \rightarrow C$ by

$$
u(z)=\sigma_{t}\left(z_{0}\right) e^{-i(\mu / \omega) t} \quad \text { for } z=\varphi_{t}\left(z_{0}\right) .
$$

From (2.15) it follows that $u$ is well defined, and from (2.12) and the properties of $\gamma$, it follows that $u$ is measurable. Now let $\widetilde{A}=\gamma^{-1}(\gamma(A))$. Then $\widetilde{A}$ is negligible. For $t \notin A$, and $s \notin \widetilde{A} \cup(\widetilde{A}-t) \cup N_{t}$ we have

$$
\begin{aligned}
u\left(\phi_{t}\left(\phi_{s}\left(z_{0}\right)\right)\right) & =u\left(\phi_{t+s}\left(z_{0}\right)\right) \\
& =\sigma_{t+s}\left(z_{0}\right) e^{-i(\mu / \omega)(t+s)} \\
& =\sigma_{t+s}\left(z_{0}\right) e^{-i(\mu / \omega) t} e^{-i(\mu / \omega) s} \\
& =\sigma_{t}\left(\phi_{s}\left(z_{0}\right)\right) \sigma_{s}\left(z_{0}\right) e^{-i(\mu / \omega) s} e^{-i(\mu / \omega) t} \\
& =\sigma_{t}\left(\phi_{s}\left(z_{0}\right)\right) u\left(\phi_{s}\left(z_{0}\right)\right) e^{-i(\mu / \omega) t} .
\end{aligned}
$$

Since almost all $z$ are of the form $z=\varphi_{s}\left(z_{0}\right)$ with $s \notin \widetilde{A} \cup(\widetilde{A}-t) \cup N_{t}$, we conclude that

$$
e^{i(\mu / \omega) t} u\left(\varphi_{t}(z)\right) \overline{u(z)}=\sigma_{t}(z) \text { for almost all } z .
$$

By $L^{p}$-continuity, we get the desired expression for $\sigma_{t}$ with $\delta=\mu / \omega$.

(2.16) Remark. Conversely, for $1 \leqslant p<\infty$, it is easy to see that if $u$ is a unimodular measurable function on $C$ and $\delta$ is any real constant, then for each $\left\{\varphi_{t}\right\}$, the family $\left\{e^{i \delta t} u\left(\varphi_{t}\right) \bar{u}\right\}$ satisfies (2.2) and (2.3).

We omit the straightforward proofs of the next two theorems.

(2.17) THEOREM. Under the hypotheses of (2.6), if $u_{1}$ and $u_{2}$ satisfy the conclusion of (2.6), then on each component of $C \backslash S, u_{1} \bar{u}_{2}$ is almost everywhere equal to a constant (depending on the component).

(2.18) THEOREM. Under the hypotheses of Theorem (2.9), if $\left(\delta_{1}, u_{1}\right)$ and $\left(\delta_{2}, u_{2}\right)$ satisfy the conclusion of (2.9), then there are a $z_{0} \in C$, a unimodular constant $\nu$, and an integer $k$ such that, denoting the angular velocity of $\left\{\phi_{t}\right\}$ by c, we have: 


$$
\text { for almost all } z \in C, \quad u_{2}(z)=u_{1}(z) v e^{i k c t}
$$

where $t$ is any real number such that $z=\varphi_{t}\left(z_{0}\right)$, and

$$
\delta_{2}=\delta_{1}-k c \text {. }
$$

(2.21) REMARK. The measure-theoretic character of (2.2) and (2.3) necessitated the detailed analysis in the proofs of Theorems (2.6) and (2.9). However, the conclusions reached in these theorems can be phrased in the language of homological algebra by saying that if $F$ is the topological (in the $L^{p}$-norm) abelian group of measurable functions $h: C \rightarrow C$ modulo equality almost everywhere made into a topological R-module by $t h=h \circ \varphi_{t}$, then in the topological group cohomology $H^{1}(\mathrm{R}, F)=0$ in the case of types (ii) and (iii), and $H^{1}(\mathrm{R}, F)$ $=C$ in the case of type (i) (see Theorem (3.1) below for the latter).

We summarize the results of this section in the following two theorems.

(2.22) THEOREM. Let $\left\{T_{t}\right\} \in \Omega_{p}, 1 \leqslant p<\infty$, and let the unique group $\left\{\varphi_{t}\right\}$ in (2.1) be of type (i). Then there are a unimodular measurable function $u$ on $C$ and real constants $\rho$ and $\delta$, with $\rho$ unique, such that for $t \in \mathbf{R}$ and $f \in$ $H^{p}\left(C^{2}\right)$

$$
\left(T_{t} f\right)(z, w)=e^{i \rho t}\left[\varphi_{t}^{\prime}(z)\right]^{1 / p} f\left(\varphi_{t}(z), e^{i \delta t} u\left(\varphi_{t}(z)\right) \overline{u(z)} w\right)
$$

$$
\text { for almost all }(z, w) \in C^{2} \text {. }
$$

Conversely, for any such $\left\{\varphi_{t}\right\}, \rho, \delta$ and $u,(2.23)$ defines a group $\left\{T_{t}\right\}$ in $\Omega_{p}$.

(2.24) THEOREM. Let $\left\{T_{t}\right\} \in \Omega_{p}, 1 \leqslant p<\infty$, and let the unique group $\left\{\varphi_{t}\right\}$ in (2.1) be of type (ii) or (iii). Then there are a unimodular measurable function $u$ on $C$ and a unique real constant $\rho$ such that for $t \in \mathbf{R}$ and $f \in$ $H^{p}\left(C^{2}\right)$,

$$
\left(T_{t} f\right)(z, w)=e^{i \rho t}\left[\varphi_{t}^{\prime}(z)\right]^{1 / p} f\left(\varphi_{t}(z), u\left(\varphi_{t}(z)\right) \overline{u(z)} w\right)
$$

$$
\text { for almost all }(z, w) \in C^{2} \text {. }
$$

Conversely, for each such $\left\{\varphi_{t}\right\}$, $\rho$ and $u,(2.25)$ defines a group $\left\{T_{t}\right\}$ in $\Omega_{p}$.

(2.26) Definition. For a group $\left\{T_{t}\right\} \in \Omega_{p}, 1 \leqslant p<\infty$,

$$
\left(T_{t} f\right)(z, w)=e^{i \rho t}\left[\varphi_{t}^{\prime}(z)\right]^{1 / p} f\left(\varphi_{t}(z), \sigma_{t}(z) w\right)
$$

we shall call the constant $\rho$, the group $\left\{\varphi_{t}\right\}$ and the family $\left\{\sigma_{t}\right\}$, respectively, the logarithmic index, the conformal group, and the cocycle of $\left\{T_{t}\right\}$. Also, we shall say that $\left\{T_{t}\right\}$ is of type (i), (ii) or (iii) according as its conformal group is. In the spirit of Remark (2.21) we also introduce the following definition. 
(2.27) Definition. Let $\left\{T_{t}\right\} \in \Omega_{p}, 1 \leqslant p<\infty$, have conformal group $\left\{\varphi_{t}\right\}$. We shall say that the cocycle $\left\{\sigma_{t}\right\}$ of $\left\{T_{t}\right\}$ is trivial if there is a unimodular measurable function $u$ on $C$ such that for each $t \in \mathrm{R}, \sigma_{t}(z)=u\left(\varphi_{t}(z)\right) \overline{u(z)}$ for almost all $z \in C$.

According to Theorem (2.24), if $\left\{T_{t}\right\}$ is of type (ii) or (iii), then $\left\{T_{t}\right\}$ has trivial cocycle. On the other hand, it is clear from (2.20) that not all groups of type (i) have trivial cocycle.

3. The infinitesimal generator of a group with trivial cocycle. In this section we are concerned with groups having trivial cocycle. This includes all groups of type (ii) and (iii). The type (i) groups with trivial cocycle can be characterized as follows.

(3.1) Theorem. Let $\left\{T_{t}\right\} \in \Omega_{p}, 1 \leqslant p<\infty$, be of type (i). Let $K$ be the set of all $\delta \in \mathbf{R}$ such that for an appropriate unimodular measurable function $u,(2.23)$ holds. Then $K$ is an element of $\mathbf{R} / c \mathbf{Z}$, where $c$ is the angular velocity of the conformal group of $\left\{T_{t}\right\}$. In particular, the cocycle of $\left\{T_{t}\right\}$ is trivial if and only if $K=c \mathbf{Z}$.

Proof. The theorem follows from Theorem (2.18) and its obvious converse.

Throughout the remainder of this section, $\left\{T_{t}\right\}$ will be a fixed element of $\Omega_{p}, 1 \leqslant p<\infty$, with generator $A$, conformal group $\left\{\phi_{t}\right\}$, and trivial cocycle $\left\{\sigma_{t}\right\}$. We pick a unimodular measurable function $u$ on $C$ such that $\sigma_{t}(z)=$ $u\left(\phi_{t}(z)\right) \overline{u(z)}$.

Since a change in the logarithmic index of $\left\{T_{t}\right\}$ only translates the generator by a scalar multiple of $I$, we shall obtain a description of $A$ under the additional assumption that the logarithmic index is zero.

Let $U$ be the isometry of $H^{p}\left(C^{2}\right)$ onto itself given by

$$
(U f)(z, w)=f(z, u(z) w) .
$$

For each $t \in R$, let $S_{t}=U T_{t} U^{-1}$. Then $S_{t}$ has the form:

$$
\left(S_{t} f\right)(z, w)=\left[\phi_{t}^{\prime}(z)\right]^{1 / p} f\left(\phi_{t}(z), w\right) \text { for } f \in H^{p}\left(C^{2}\right) .
$$

Clearly $\left\{S_{t}\right\} \in \Omega_{p}$. Denote the generator of $\left\{S_{t}\right\}$ by A. Thus the trivial cocycle case reduces to the case $\sigma_{t} \equiv 1$ for $t \in \mathbf{R}$, and we proceed now to obtain an expression for $A$.

Let $f \in D(A)$. Without loss of generality we assume that $f$ and $A f$ are Borel measurable and all their sections are integrable. Let $q$ denote the invariance polynomial of $\left\{\phi_{t}\right\}$, and $S$ the set of common fixed points in $\mathbf{C}_{e}$ of $\left\{\phi_{t}\right\}$. Then by $\left[2,(1.6)\right.$ and (1.7)] for each $z \in \mathbf{C}, q(z)=\left.(d / d t)\right|_{t=0} \phi_{t}(z)$, and $S \cap \mathbf{C}$ consists of the zeros of $q$. 
With differentiation at $t=0$ in the $L^{p}\left(C^{2}\right)$-sense, we have

$$
\begin{aligned}
\left.\frac{d}{d t}\right|_{t=0} f\left(\varphi_{t}(z), w\right) & =\left.\frac{d}{d t}\right|_{t=0}\left\{\left[\varphi_{t}^{\prime}(z)\right]^{-1 / p} S_{t} f\right\} \\
& =A f-\left(\frac{1}{p}\right) q^{\prime}(z) f
\end{aligned}
$$

hence

$$
\begin{aligned}
\left.\frac{d}{d t}\right|_{t}=0 & \left\{\left|\varphi_{t}^{\prime}(z)\right| f\left(\varphi_{t}(z), w\right)\right\} \\
= & A f-p^{-1} q^{\prime}(z) f+\left[q^{\prime}(z)-z^{-1} q(z)\right] f \\
& =A f+\left(1-p^{-1}\right) q^{\prime}(z) f-z^{-1} q(z) f .
\end{aligned}
$$

Denote by $h$ the Borel measurable function on the right-hand side of (3.3). Note that $h \in L^{p}\left(C^{2}\right)$ and has all its sections integrable. For any two points $\xi_{0}, \xi_{1} \in C$, let $\left[\xi_{0}, \xi_{1}\right]$ denote the closed counterclockwise arc of $C$ from $\xi_{0}$ to $\xi_{1}$ (if $\xi_{0}=\xi_{1}$, we take $\left[\xi_{0}, \xi_{1}\right]$ to be $\left\{\xi_{0}\right\}$ ). The symbol $\int_{\xi_{0}}^{\xi_{1}}$ will stand for $\int_{\left[\xi_{0}, \xi_{1}\right]}$. By virtue of (3.3) we have for $z_{0}, z_{1}, w_{0} \in C$,

$$
\begin{aligned}
\lim _{t \rightarrow 0} \int_{z_{0}}^{z_{1}} d \lambda(z) \int_{1}^{w_{0}} t^{-1}\left\{\left|\varphi_{t}^{\prime}(z)\right| f\left(\varphi_{t}(z), w\right)\right. & -f(z, w)\} d \lambda(w) \\
& =\int_{z_{0}}^{z_{1}} d \lambda(z) \int_{1}^{w_{0}} h(z, w) d \lambda(w) .
\end{aligned}
$$

Thus, as $t \rightarrow 0$,

$$
\begin{aligned}
t^{-1} \int_{\varphi_{t}\left(z_{0}\right)}^{\varphi_{t}\left(z_{1}\right)} d \lambda(z) \int_{1}^{w_{0}} f(z, w) d \lambda(w) & \\
& -t^{-1} \int_{z_{0}}^{z_{1}} d \lambda(z) \int_{1}^{w_{0}} f(z, w) d \lambda(w)
\end{aligned}
$$

approaches $\int_{z_{0}}^{z_{1}} d \lambda(z) \int_{1}^{w_{0}} h(z, w) d \lambda(w)$.

Enumerate the rational numbers in $[0,2 \pi]$ in a sequence $\left\{r_{k}\right\}$. Then there is a set $N$ of measure 0 in $[0,2 \pi)$ such that for each $\theta \in[0,2 \pi) \backslash N$

$$
\frac{d}{d \theta} \int_{0}^{\theta} d v \int_{0}^{r_{k}} f\left(e^{i v}, e^{i \psi}\right) d \psi=\int_{0}^{r_{k}} f\left(e^{i \theta}, e^{i \psi}\right) d \psi
$$

for all $k$.

Fix permanently a number $\theta_{0} \in[0,2 \pi) \backslash N$. In (3.4) take $z_{0}=e^{i \theta} 0$ and $z_{1}=e^{i \theta_{1}}$ where $\theta_{1} \in[0,2 \pi) \backslash N, \theta_{1} \neq \theta_{0}$. Also take $w_{0}$ in (3.4) to be of the form $e^{i r_{k}}$. Clearly, there is an analytic logarithm $L$ defined on the complement of a ray from the origin such that for all sufficiently small values of $|t|,\left[\varphi_{t}\left(z_{0}\right)\right.$, $\left.\varphi_{t}\left(z_{1}\right)\right]$ is contained in the domain of $L$. Let $a_{0}(t)=\operatorname{Im}\left[L\left(\varphi_{t}\left(z_{0}\right)\right)\right], a_{1}(t)=$ $\operatorname{Im}\left[L\left(\varphi_{t}\left(z_{1}\right)\right)\right]$. It now follows by (3.4) and obvious manipulation that 


$$
\begin{aligned}
\int_{z_{0}}^{z_{1}} d \lambda(z) & \int_{0}^{r_{k}} h\left(z, e^{i \psi}\right) d \psi \\
& =\lim _{t \rightarrow 0}\left\{(2 \pi t)^{-1} \int_{a_{1}(0)}^{a_{1}(t)} d v \int_{0}^{r_{k}} f\left(e^{i v}, e^{i \psi}\right) d \psi\right. \\
& \left.-(2 \pi t)^{-1} \int_{a_{0}(0)}^{a_{0}(t)} d v \int_{0}^{r_{k}} f\left(e^{i v}, e^{i \psi}\right) d \psi\right\} .
\end{aligned}
$$

For $j=0,1$ we have an integer $n_{j}$ such that

$$
a_{j}(0)=\theta_{j}+2 n_{j} \pi
$$

By the chain rule,

$$
\begin{aligned}
\lim _{t \rightarrow 0} t^{-1} \int_{a_{j}(0)}^{a_{j}(t)} d v \int_{0}^{r} f\left(e^{i v}, e^{i \psi}\right) d \psi \\
=\left[\lim _{t \rightarrow 0}\left\{t^{-1} \int_{a_{j}(0)}^{a_{j}(0)+t} d v \int_{0}^{r} f\left(e^{i v}, e^{i \psi}\right) d \psi\right\}\right] a_{j}^{\prime}(0) .
\end{aligned}
$$

By virtue of (3.7), (3.5) and the fact that

$$
a_{j}^{\prime}(0)=-\left.i \frac{d}{d t}\right|_{t=0} L\left(\phi_{t}\left(z_{j}\right)\right)=\left(i z_{j}\right)^{-1} q\left(z_{j}\right),
$$

we see that the right-hand side of (3.8) equals $\left(i z_{j}\right)^{-1} q\left(z_{j}\right) \int_{0}^{r} k f\left(z_{j}, e^{i \psi}\right) d \psi$.

Now (3.6) can be written:

$$
\begin{aligned}
& 2 \pi \int_{z_{0}}^{z_{1}} d \lambda(z) \int_{0}^{r} h\left(z, e^{i \psi}\right) d \psi \\
& =\left(i z_{1}\right)^{-1} q\left(z_{1}\right) \int_{0}^{r} f\left(z_{1}, e^{i \psi}\right) d \psi-\left(i z_{0}\right)^{-1} q\left(z_{0}\right) \int_{0}^{r k} f\left(z_{0}, e^{i \psi}\right) d \psi .
\end{aligned}
$$

After interchanging the order of integration on the left of (3.9) and making use of the density of $\left\{r_{k}\right\}$ in $[0,2 \pi]$, we see that for almost all $\xi \in C$, the following equation holds for almost all $w \in C$.

(3.10) $2 \pi \int_{z_{0}}^{\zeta} h(z, w) d \lambda(z)=(i \zeta)^{-1} q(\zeta) f(\zeta, w)-\left(i z_{0}\right)^{-1} q\left(z_{0}\right) f\left(z_{0}, w\right)$.

From (3.3) we have:

$$
\begin{aligned}
\lim _{t \rightarrow 0}|t|^{-1} \int\left|\int\left[\left|\phi_{t}^{\prime}(z)\right| f\left(\phi_{t}(z), w\right)-f(z, w)\right] d \lambda(z)\right| d \lambda(w) & \\
= & \int\left|\int h(z, w) d \lambda(z)\right| d \lambda(w) .
\end{aligned}
$$

The inner integral on the left of (3.11) vanishes identically. Hence there is a negligible subset $M$ of $C$ such that $\operatorname{Sh}(z, w) d \lambda(z)=0$ for $w \in C M$. It follows 
that if $w \in C \backslash M$, then for all $\xi, \zeta, \eta \in C$ :

$$
\int_{\xi}^{\xi} h(z, w) d \lambda(z)=\int_{\xi}^{\eta} h(z, w) d \lambda(z)+\int_{\eta}^{\zeta} h(z, w) d \lambda(z) .
$$

Next, let $g$ be a Borel measurable function on $C^{2}$ such that for all $\zeta \in C \backslash S, w$ $\in C$

$$
g(\zeta, w)=2 \pi i \zeta(1 / q(\zeta))\left\{\int_{z_{0}}^{\zeta} h(z, w) d \lambda(z)+\left(2 \pi i z_{0}\right)^{-1} q\left(z_{0}\right) f\left(z_{0}, w\right)\right\} .
$$

By (3.10) $f=g$ almost everywhere on $C^{2}$ (hence, in particular, $g \in D(A)$ ).

Suppose now that $\left\{\phi_{t}\right\}$ is of type (ii) or (iii). Let $\alpha \in S$. For all $w \in C W$, $g(\zeta, w)=2 \pi i \zeta(1 / q(\zeta))$

$$
\cdot\left\{\int_{\alpha}^{\zeta} h(z, w) d \lambda(z)+\int_{z_{0}}^{\alpha} h(z, w) d \lambda(z)+\left(2 \pi i z_{0}\right)^{-1} q\left(z_{0}\right) f\left(z_{0}, w\right)\right\}
$$

for $\zeta \in C S$.

For $z \in C$, let $\theta=\arg z, \arg \alpha \leqslant \theta<\arg \alpha+2 \pi$. Let $E$ be a component of $C \backslash S$, and let a (resp., $b)$ equal $\inf \{\theta: z \in E\}$ (resp., $\sup \{\theta: z \in E\})$.

If $w \in C \backslash M$, then by $(3.12) g\left(e^{i \theta}, w\right)$ is an absolutely continuous function of $\theta$ on each closed subinterval of $(a, b)$, and we have for almost all $\theta \in(a, b)$

$$
q\left(e^{i \theta}\right) \frac{d g\left(e^{i \theta}, w\right)}{d \theta}+i e^{i \theta} q^{\prime}\left(e^{i \theta}\right) g\left(e^{i \theta}, w\right)=i e^{i \theta} h\left(e^{i \theta}, w\right)+i q\left(e^{i \theta}\right) g\left(e^{i \theta}, w\right)
$$

If we combine this last equation with the definition of $h$ and the fact that $f=g$ almost everywhere on $C^{2}$, we get after some simplification that for almost all $w \in C: g\left(e^{i \theta}, w\right)$ is an absolutely continuous function of $\theta$ on each closed subinterval of $(a, b)$, and for almost all $\theta \in(a, b)$

$$
(-i) e^{-i \theta} q\left(e^{i \theta}\right) \frac{d g\left(e^{i \theta}, w\right)}{d \theta}+p^{-1} q^{\prime}\left(e^{i \theta}\right) g\left(e^{i \theta}, w\right)=(A g)\left(e^{i \theta}, w\right) .
$$

If $\left\{\phi_{t}\right\}$ is of type (i), then for $w \in C \backslash M$, the equation (3.12) (with $\alpha$ in (3.12) replaced by 1 ) holds for all $\zeta \in C$. Thus for $w \in C \backslash M$, we get

$$
\begin{aligned}
g\left(e^{i \theta}, w\right)= & 2 \pi i e^{i \theta}\left(1 / q\left(e^{i \theta}\right)\right) \\
& \cdot\left\{(2 \pi)^{-1} \int_{0}^{\theta} h\left(e^{i v}, w\right) d v+\int_{z_{0}}^{1} h(z, w) d \lambda(z)\right.
\end{aligned}
$$

$$
\begin{aligned}
\left.+\left(2 \pi i z_{0}\right)^{-1} q\left(z_{0}\right) f\left(z_{0}, w\right)\right\} \\
\text { for } 0 \leqslant \theta \leqslant 2 \pi .
\end{aligned}
$$


By reasoning similar to the type (ii) and type (iii) cases, we now get that for almost all $w \in C: g\left(e^{i \theta}, w\right)$ is an absolutely continuous function of $\theta$ on $[0,2 \pi]$, and (3.13) holds for almost all $\theta \in[0,2 \pi]$.

(3.15) Definition. We define the linear transformation $B$ as follows. If $\left\{\phi_{t}\right\}$ is of type (i) (resp., of type (ii) or (iii)), the domain of $B, D(B)$, consists of all Borel measurable $F \in H^{p}\left(C^{2}\right)$ for which there is a Borel measurable $G \in H^{p}\left(C^{2}\right)$ such that for almost all $w \in C: F(z, w)$ is an absolutely continuous function of $z$ on $C$ (resp., on each closed subinterval of each component of $C \backslash S$ ), and, on $C$ (resp., on each component of $C \backslash S$ ),

$$
\begin{aligned}
(-i) e^{-i \theta} q\left(e^{i \theta}\right) \frac{d F\left(e^{i \theta}, w\right)}{d \theta} & +p^{-1} q^{\prime}\left(e^{i \theta}\right) F\left(e^{i \theta}, w\right) \\
& =G\left(e^{i \theta}, w\right) \text { for almost all } \theta .
\end{aligned}
$$

For $F, G$ as above, we define $B F=G$.

It is easy to see that $B$ in Definition (3.15) is a well-defined linear operator which extends $A$. In fact we have

(3.17) THEOREM. $B=A$.

Proof. Since the spectrum of $A$ is a subset of $i R$, while $B$ extends $A$, it suffices to show that for some nonzero real number $r,(B-r I)$ is one-to-one.

Suppose $\left\{\phi_{t}\right\}$ is of type (i), and $B F=p^{-1} F$, where for all $w$ outside some negligible subset $N$ of $C, F\left(e^{i \theta}, w\right)$ is an absolutely continuous function of $\theta$ on $[0,2 \pi]$, and for $w \in C \backslash N$

$$
\begin{aligned}
& (-i) e^{-i \theta} q\left(e^{i \theta}\right) \frac{d F\left(e^{i \theta}, w\right)}{d \theta}+p^{-1} q^{\prime}\left(e^{i \theta}\right) F\left(e^{i \theta}, w\right) \\
& =p^{-1} F\left(e^{i \theta}, w\right), \text { for almost all } \theta \in[0,2 \pi] .
\end{aligned}
$$

It follows from absolute continuity that (3.18) holds for all $\theta \in[0,2 \pi]$, and so, by elementary means we get that for each $w \in C \backslash N$, there is a complex constant $K_{w}$ such that for $\theta \in[0,2 \pi]$

$$
F\left(e^{i \theta}, w\right)=K_{w} \exp \left\{-p^{-1} \int_{0}^{\theta}\left[\frac{\left(q^{\prime}\left(e^{i v}\right)-1\right)}{q\left(e^{i v}\right)}\right] i e^{i v} d v\right\},
$$

where "exp" stands for "exponential of". If $K_{w} \neq 0$, then equating the values at $\theta=0$ and $\theta=2 \pi$ of the right-hand side of (3.19) gives the existence of an integer $k$ such that:

$$
(2 \pi i)^{-1} \int_{C}\left(q^{\prime}(z)-1\right)(1 / q(z)) d z=k p
$$

the integral in (3.20) being a contour integral. By the argument principle, 
$(2 \pi i)^{-1} \int_{C}\left(q^{\prime}(z) / q(z)\right) d z=1$. The expression for $q$ in [1, Theorem (1.6)-(i)] allows us to conclude by the residue theorem that $(2 \pi i)^{-1} \int_{C}(1 / q(z)) d z=-i c^{-1}$, where $c$ is the angular velocity of $\left\{\phi_{t}\right\}$. Substitution in (3.20) gives the absurd conclusion that $1+i c^{-1}=k p$. Hence $K_{w}=0$, and so $F=0$ almost everywhere on $C^{2}$. This concludes the proof of the type (i) case.

Now let $\left\{\varphi_{t}\right\}$ be of type (ii) with common fixed point $\alpha$ and the representation [1, (1.8)], and suppose that for the nonzero real constant $c$ in that representation we have $B F=c F$, where, in a fashion similar to the foregoing, there is a negligible subset $M$ of $C$ such that for $w \in C \backslash M, F(; w) \in L^{p}(d \lambda)$ and

$$
\begin{array}{r}
q\left(e^{i \theta}\right) \frac{d F\left(e^{i \theta}, w\right)}{d \theta}+i e^{i \theta}\left(p^{-1} q^{\prime}\left(e^{i \theta}\right)-c\right) F\left(e^{i \theta}, w\right)=0 \\
\text { for } \arg \alpha<\theta<\arg \alpha+2 \pi
\end{array}
$$

Define $f$ on $C \backslash\{\alpha\}$ by the equation

$$
f(z)=(z-\alpha)^{2\left(p^{-1}-1\right)} \exp \{(-i \alpha) /(z-\alpha)\} .
$$

If $w \in C \backslash M$, it is straightforward to verify that for $\arg \alpha<\theta<\arg \alpha+2 \pi$ the product of $f\left(e^{i \theta}\right)$ and the left-hand side of (3.21) is $d f\left(e^{i \theta}\right) q\left(e^{i \theta}\right) F\left(e^{i \theta}, w\right) / d \theta$. So we conclude that for $w \in C \backslash M$, there is a constant $K_{w}$ such that

$$
F\left(e^{i \theta}, w\right)=K_{w} /\left[f\left(e^{i \theta}\right) q\left(e^{i \theta}\right)\right] \text { for } \arg \alpha<\theta<\arg \alpha+2 \pi .
$$

It is easy to see that $(f q)^{-1} \notin L^{p}(d \lambda)$. This establishes the desired conclusion in the type (ii) case.

To conclude the proof, suppose $\left\{\phi_{t}\right\}$ is of type (iii), with the representation $[1,(1.9)]$ (in particular, $S=\{\alpha, \beta\}$ ), and suppose that for the positive constant $c$ of that representation we have $B F=c F$, where, for all unimodular $w$ outside some negligible subset $M$ of $C, F(\cdot, w) \in L^{p}(d \lambda)$, and, on each component of $C \backslash S,(3.21)$ holds for all $\theta$. Define the function $f$ on $C \backslash S$ by the equation $f(z)=(z-\alpha)^{(1 / p)-2}(z-\beta)^{1 / p}$. Then, just as in the type (ii) case, $f\left(e^{i \theta}\right)$ is an integrating factor for (3.21). It follows that for each $w \in C \backslash M$, and each component $E$ of $C \backslash S$, there is a constant $K_{w, E}$, such that $F(z, w)=K_{w, E} /[f(z) q(z)]$ for $z \in E$. It is easy to see that in this case $(f q)^{-1} \notin L^{p}(E)$. The proof of the theorem is complete.

We summarize the results of this section in the following theorem.

(3.22) THEOREM. Let $\left\{T_{t}\right\} \in \Omega_{p}, 1 \leqslant p<\infty$, have conformal group $\left\{\phi_{t}\right\}$, logarithmic index $\rho$, and trivial cocycle $\left\{\sigma_{t}\right\}$. If $u$ is any unimodular measurable function on $C$ such that for all $t \in \mathbf{R}, \sigma_{t}=u\left(\phi_{t}\right) \bar{u}$, let $U$ be the isometry of $H^{p}\left(C^{2}\right)$ onto itself in (3.2). Then $\left\{U T_{t} U^{-1}\right\} \in \Omega_{p}$ and has generator $(B+$ $i \rho I)$, where $B$ is the operator defined in (3.15). 
4. The infinitesimal generator of a group with nontrivial cocycle. Let $\left\{T_{t}\right\} \in \Omega_{p}, 1 \leqslant p<\infty$, be a group of type (i) of the form

$$
\left(T_{t} f\right)(z, w)=\left[\varphi_{t}^{\prime}(z)\right]^{1 / p} f\left(\varphi_{t}(z), e^{i \delta t} u\left(\varphi_{t}(z)\right) \overline{u(z)} w\right) .
$$

Let $c$ be the angular velocity and $\tau$ the common fixed point in $D$ of the group $\left\{\varphi_{t}\right\}$.

The case of trivial cocycle (that is, $(\delta / c) \in \mathrm{Z}$ ) is already covered by $\S 3$, but the method that we shall employ here, based on multipliers on the Fourier coefficients, applies to the type (i) case without additional assumptions. For $f \in L^{p}\left(C^{2}\right)$, we denote by $\left\{\hat{f}_{k, j}\right\}_{k, j=-\infty}^{\infty}$ the double sequence of Fourier coefficients of $f$.

Let $\mu$ be the Möbius transformation of $D$ defined by $\mu(z)=(z-\tau) /$ $(\bar{\gamma} z-1)$. Then (see [2, Theorem (1.10)]) $\varphi_{t}(z)=\mu\left(e^{i c t} \mu(z)\right)$, and $\mu$ is its own inverse map. On $\bar{D}$ a continuous logarithm for $\mu^{\prime}$ is given by:

$$
L(z)=-2\{\log |\bar{\tau} z-1|+i \arg (\bar{\tau} z-1)\}+\log \left(1-|\tau|^{2}\right)+3 \pi i,
$$

where $0<\arg (\bar{\tau} z-1)<2 \pi$. We define $\left[\mu^{\prime}(z)\right]^{1 / p}$ to be $\exp \{L(z) / p\}$. With this standardization of $\left(\mu^{\prime}\right)^{1 / p}$, we define the isometry $V$ of $H^{p}\left(C^{2}\right)$ onto itself as follows:

$$
(V f)(z, w)=\left[\mu^{\prime}(z)\right]^{1 / p} f(\mu(z), w) .
$$

It is easy to see that $V^{-1}=e^{-2 \pi i / p} V$. Further, let $U$ be the isometry of $H^{p}\left(C^{2}\right)$ onto itself given by $(U f)(z, w)=f(z, u(z) w)$, and put

$$
W=V^{-1} U
$$

For each $t \in \mathbf{R}$, let $S_{t}=e^{-i c t / p} W T_{t} W^{-1}$. It is straightforward to check that

$$
\left(S_{t} f\right)(z, w)=f\left(e^{i c t} z, e^{i \delta t} w\right) \text { for } f \in H^{p}\left(C^{2}\right) .
$$

Clearly, $\left\{S_{t}\right\} \in \Omega_{p}$. Let $A$ be the generator of $\left\{S_{t}\right\}$, and let $\tilde{\lambda}$ be normalized Lebesgue measure on $C^{2}$. If $f \in D(A)$, then for all $m, n \in \mathbf{Z}$,

$$
\begin{aligned}
t^{-1} \int\left[f\left(e^{i c t} z, e^{i \delta t} w\right)-f(z, w)\right] z^{-m} w^{-n} d \tilde{\lambda}(z, w) \\
=t^{-1}\left[e^{i m c t} e^{i n \delta t}-1\right] \int f(z, w) z^{-m} w^{-n} d \tilde{\lambda}(z, w)
\end{aligned}
$$

Letting $t \rightarrow 0$ in (4.2), we get for all $m \in \mathbf{Z}, n \in \mathbf{Z},(\widehat{A f f})_{m, n}=i(m c+n \delta) \hat{f}_{m, n}$.

(4.3) Definition. We define the operator $G$ as follows. $D(G)$ consists of all $f \in H^{p}\left(C^{2}\right)$ such that for some $g \in L^{p}\left(C^{2}\right), \hat{g}_{m, n}=i(m c+n \delta) \hat{f}_{m, n}$ for all $m \in \mathbf{Z}, n \in \mathbf{Z}$ (such a $g$ is necessarily a uniquely determined element of $\left.H^{p}\left(C^{2}\right)\right)$. We then set $G f=g$. 
Clearly, $G$ is a linear operator which extends $A$. It is easy to see that if $G f=f$, then $f=0$. Thus $G=A$.

We summarize the foregoing in the following theorem.

(4.4) THEOREM. Let $\left\{T_{t}\right\} \in \Omega_{p}, 1 \leqslant p<\infty$, be a group of type (i) having the form

$$
\left(T_{t} f\right)(z, w)=e^{i \rho t}\left[\varphi_{t}^{\prime}(z)\right]^{1 / p} f\left(\varphi_{t}(z), e^{i \delta t} u\left(\varphi_{t}(z) \overline{u(z)} w\right) \text { for } f \in H^{p}\left(C^{2}\right) .\right.
$$

Let $W$ be the isometry in (4.1). Then $\left\{W T_{t} W^{-1}\right\}$ is a type (i) group with generator $G+i(\rho+(c / p)) I$ where $G$ is the operator defined in (4.3).

We close by showing that if $\delta / c$ is an irrational number, then the domain of the infinitesimal generator $G$ of the group $\left\{S_{t}\right\}$ in this section contains a function $f$ which has neither a weak partial derivative with respect to $\theta$ nor a weak partial derivative with respect to $\psi$ in the sense that for no $G \in L^{1}\left(C^{2}\right)$ does either of the following equations hold identically for all $C^{1}$-functions $h\left(e^{i \theta}, e^{i \psi}\right)$ of $\theta, \psi$ :

$$
\begin{aligned}
& \int h G d \tilde{\lambda}=-\int f \frac{\partial h}{\partial \theta} d \tilde{\lambda} \\
& \int h G d \tilde{\lambda}=-\int f \frac{\partial h}{\partial \psi} d \tilde{\lambda} .
\end{aligned}
$$

In fact, $\{m c+n \delta: m \in \mathbf{Z}, n \in \mathbf{Z}, n>0\}$ is dense in $\mathbf{R}$, since $\delta / c$ is irrational. It is easy to see from this that there are two sequences of integers $\left\{m_{k}\right\}_{k=1}^{\infty},\left\{n_{k}\right\}_{k=1}^{\infty}$, each with distinct terms, such that $\left(m_{k} c+n_{k} \delta\right) \rightarrow 0$, and, for all $k,\left|m_{k}\right|>k^{4}, n_{k}>k^{4}$. Thus the series $\Sigma_{k=1}^{\infty} k^{-2} z^{m} w^{n_{k}}$ (resp., $\left.\sum_{k=1}^{\infty} i\left(m_{k} c+n_{k} \delta\right) k^{-2} z^{m} k_{w^{n}}{ }^{n}\right)$ converges uniformly on $C^{2}$ to a continuous function $f$ (resp., $g$ ) in $H^{p}\left(C^{2}\right)$. Clearly $G f=g$. However, if we take $h=$

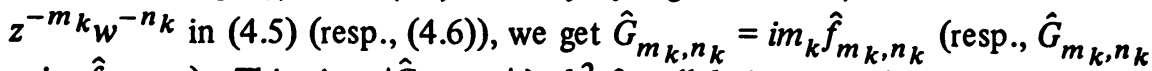
$\left.=i n_{k} \hat{f}_{m_{k}, n_{k}}\right)$. This gives $\left|\hat{G}_{m_{k}, n_{k}}\right|>k^{2}$ for all $k$, in contradiction to the Riemann-Lebesgue lemma.

\section{REFERENCES}

1. E. Berkson, R. Kaufman and H. Porta, Möbius transformations of the disc and one-parameter groups of isometries of $H^{p}$, Trans. Amer. Math. Soc. 199 (1974), 223-239.

2. E. Berkson and H. Porta, Hermitian operators and one-parameter groups of isometries in Hardy spaces, Trans. Amer. Math. Soc. 185 (1973), 331-344. MR 49 \#3597.

3. J. L. Doob, Stochastic processes, Wiley, New York; Chapman \& Hall, London 1953. MR 15, 445.

4. N. Dunford and J. T. Schwartz, Linear operators. I: General theory, Pure ana Appl. Math., vol. 7, Interscience, New York, 1958. MR 22 \#8302.

5. N. Lal and S. Merrill, Isometries of $H^{p}$ spaces of the torus, Proc. Amer. Math. Soc. 31 (1972), 465-471. MR 45 \#5735.

DEPARTMENT OF MATHEMATICS, UNIVERSITY OF ILLINOIS, URBANA, ILLINOIS 61801 